\section{Subretinal Tissue Plasminogen Ac- tivator, Bevacizumab and Partial Gas Tamponade for 4-Week-old Submacular Hemorrhage in Reti- nal Arterial Macroaneurysm}

\author{
Dear Editor,
}

Retinal arterial macroaneurysms (RAMs) are focal, aneurysmal changes in the retinal artery. Visual outcomes of RAMs vary with its location and complications. A common complication of RAM is rupture of the microaneurysm which may lead to hemorrhage at various anatomical locations, such as preretinal hemorrhages, sub-internal limiting membrane hemorrhages and intraretinal and subretinal hemorrhages, resulting in acute visual disturbances [1]. Submacular hemorrhage is toxic to photoreceptor cells, and its visual prognosis is generally guarded. The results of submacular hemorrhage treatment with subretinal injections of recombinant tissue plasminogen activator (rt-PA) with or without a full gas tamponade have been reported [2-4]. This is the first report of a patient with significantly improved vision after subretinal rt-PA, bevacizumab, and partial gas tamponade in a case involving a 4-week-old submacular hemorrhage secondary to RAM.

A 69-year-old man on aspirin and clopidogrel treatment visited our hospital with a complaint of decreased visual acuity in the right eye for one week. His best-corrected visual acuity (BCVA) was 20 / 200 in the right eye. Slit-lamp examination revealed no anterior segment abnormalities. Funduscopic examination showed a 3.2-disc diameter (horizontal) $\times 2.3$-disc diameter (vertical) sized, macula-involving, subretinal hemorrhage and preretinal hemorrhage, presumably originating from an inferotemporal RAM rupture (Fig. 1A-1D). To displace the subretinal hemorrhage from the macula, we injected bevacizumab $(0.04$ $\mathrm{mL})$ and $\mathrm{C}_{3} \mathrm{~F}_{8}$ pure gas $(0.3 \mathrm{~mL})$ intravitreally and placed

Received: November 12, 2019 Final revision: December 28, 2019 Accepted: January 9, 2020 the patient in the prone position.

At a follow-up examination three weeks later, the submacular hemorrhage had not been displaced, and BCVA in his right eye had deteriorated to $20 / 500$. After informing the patient about the benefits and risks of surgical treatment, we performed a 3-port pars plana vitrectomy with 23 -gauge instruments. After vitrectomy and internal limiting membrane peeling to evacuate the sub-internal limiting membrane hemorrhage (Fig. 1E), rt-PA (12.5 $\mu \mathrm{g} / 0.1$ $\mathrm{mL})$ and bevacizumab $(2.5 \mathrm{mg} / 0.1 \mathrm{~mL})$ were injected in the subretinal hemorrhage using a 40 -gauge needle tip until the bleb extended over the inferior equator of the fundus (Fig. 1F). Fluid-air exchange and partial gas tamponade with $18 \% \mathrm{SF}_{6}$ were performed, and the patient was instructed to remain in the sitting position.

One week later, a vitreous hemorrhage appeared. Because no other specific findings were discovered on ultrasonography, he was scheduled for regular follow-up visits with no interventions. Five weeks later, the vitreous hemorrhage had absorbed, and a faint subretinal hemorrhage around the ruptured macroaneurysm was noted. Seven months after surgery, the BCVA of the right eye had improved to $20 / 30$, and fundus examination revealed contracted RAM and resorbed subretinal hemorrhage (Fig. $1 \mathrm{G}-1 \mathrm{~J})$.

Herein, we reported a patient with a subretinal hemorrhage due to ruptured RAM with a favorable visual outcome after a sequential combination of treatment modalities. Subretinal injections of rt-PA, a serine protease, may have helped resorb and evacuate the subretinal blood clot, which had lasted for over 4 weeks near the fovea. Bevacizumab, a monoclonal antibody and an angiogenesis inhibitor, augments the treatment of RAM and associated complications by stabilizing the blood-retinal barrier, decreasing vascular permeability and contracting the vessels [5]. In the present case, intravitreal bevacizumab, injected one week after symptom onset, and subretinal bevacizumab, injected during vitrectomy, may have contributed to successful resolution of the submacular hemorrhage and favorable visual outcomes. Although we have performed subretinal bevacizumab injections intraoperatively, the effect of intravitreal and subretinal bevacizumab injections might be similar because bevacizumab can cross through 

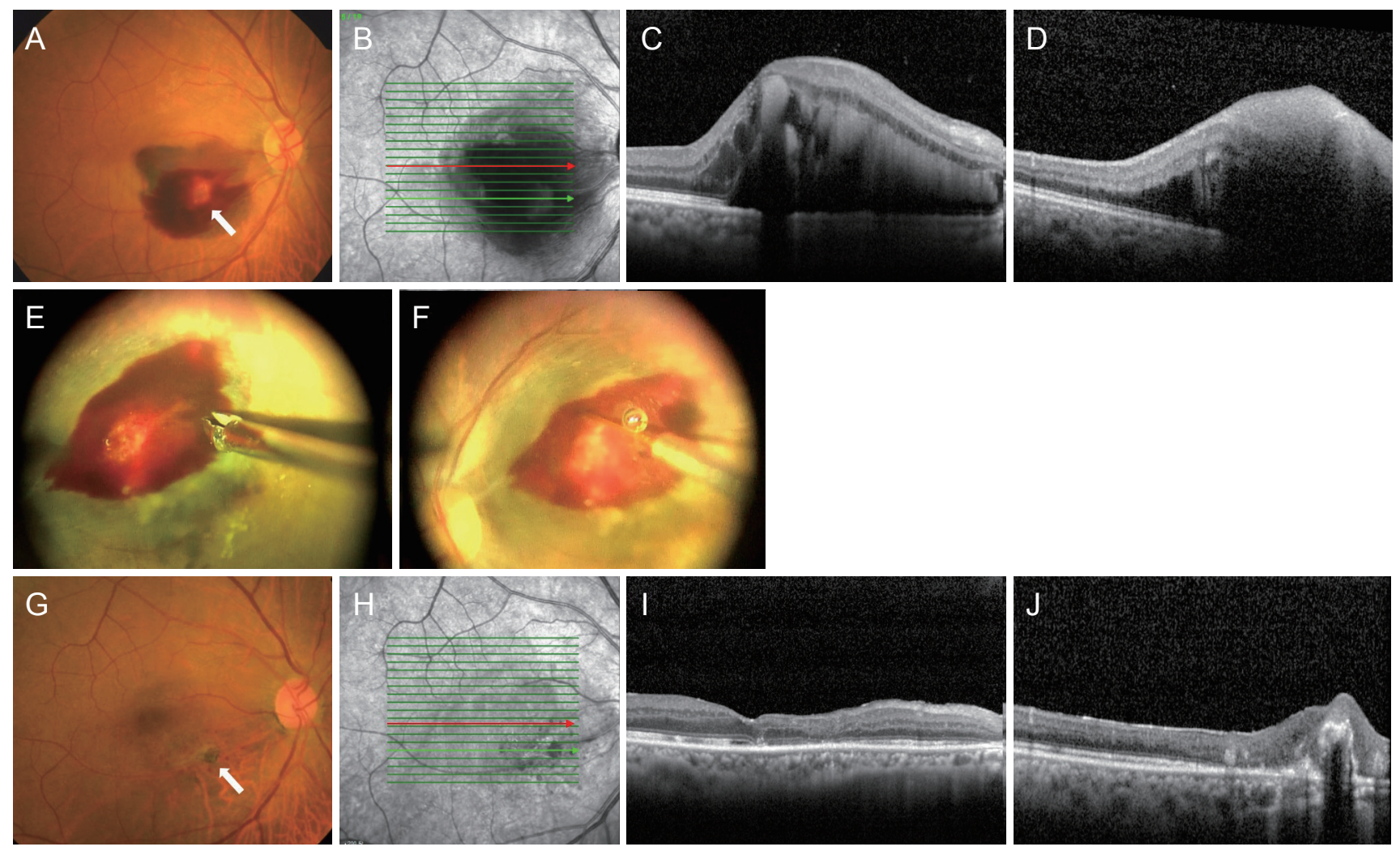

Fig. 1. Preoperative and postoperative fundus photographs and infrared images with overlaid raster scans (red and green lines) showing their positions in the spectral domain optical coherence tomography (OCT). (A) Preoperative fundus photograph demonstrated a subretinal hemorrhage around a retinal macroaneurysm (white arrow). (B) Preoperative infrared image with overlaid raster scans. (C) Preoperative OCT (horizontal raster) showed the fovea-involving intraretinal and subretinal hemorrhages. (D) Preoperative OCT (horizontal raster) crossing the macroaneurysm site with the sub-internal limiting membrane and the subretinal hemorrhage. (E) Intraoperative fundus photograph demonstrated peeling of the internal limiting membrane with end-gripping forceps. (F) Intraoperative fundus photograph showed recombinant tissue plasminogen activator $(12.5 \mu \mathrm{g} / 0.1 \mathrm{~mL})$ and bevacizumab $(2.5 \mathrm{mg} / 0.1 \mathrm{~mL})$ injected in the subretinal space using a 40-gauge needle tip. (G) Postoperative fundus photo at 7 months revealed a resorbed subretinal hemorrhage and contracted scarchanged retinal macroaneurysm (white arrow). (H) Postoperative infrared image at 7 months with overlaid raster scans. (I) Postoperative OCT (horizontal raster) at 7 months depicted a localized photoreceptor disruption at the previous subretinal hemorrhage site. (J) Postoperative OCT (horizontal raster) at 7 months revealed an elevated macroaneurysm lesion with some surrounding exudates.

the retina, and a retinal macroaneurysm is usually located at the inner layer of retina as well. In addition, considering the inferior location of the subretinal hemorrhage, we injected rt-PA and bevacizumab downward so that the subretinal bleb could extend to the inferior retinal periphery. Utilizing a partial gas tamponade and maintaining the patient in the sitting position helped avoid upward relocation of the subretinal hemorrhage. The resorbed hemorrhage may have evacuated into the vitreous cavity, resulting in the postoperative vitreous hemorrhage. To the best of our knowledge, the final visual outcome in this case was more favorable than that reported in other cases of the fovea in- volving, a relatively old subretinal hemorrhage after RAM rupture [2]. In selected cases, subretinal rtPA and bevacizumab injections with partial gas tamponade may help in the treatment of old submacular hemorrhages secondary to RAMs.

Sooyeon Choe, Baek-Lok Oh

Department of Ophthalmology, Seoul National University Hospital, Seoul National University College of Medicine, Seoul, Korea

E-mail (Baek-LokOh): baeklok@snu.ac.kr 


\section{Conflict of Interest}

No potential conflict of interest relevant to this article was reported.

\section{References}

1. Rabb MF, Gagliano DA, Teske MP. Retinal arterial macroaneurysms. Surv Ophthalmol 1988;33:73-96.

2. Inoue M, Shiraga F, Shirakata Y, et al. Subretinal injection of recombinant tissue plasminogen activator for submacular hemorrhage associated with ruptured retinal arterial macroaneurysm. Graefes Arch Clin Exp Ophthalmol 2015;253:1663-9.

3. Wu TT, Sheu SJ. Intravitreal tissue plasminogen activator and pneumatic displacement of submacular hemorrhage secondary to retinal artery macroaneurysm. J Ocul Pharmacol Ther 2005;21:62-7.

4. Sonmez K, Ozturk F, Ozcan PY. Treatment of multilevel macular hemorrhage secondary to retinal arterial macroaneurysm with submacular tissue plasminogen activator. Eur J Ophthalmol 2012;22:1026-31.

5. Golan S, Goldenberg D, Goldstein M. Long-term follow-up of intravitreal bevacizumab in retinal arterial macroaneurysm: a case report. Case Rep Ophthalmol 2011;2:387-91. 